\title{
WEAK COVERING WITHOUT COUNTABLE CLOSURE
}

\author{
W. J. Mitchell And E. SChimmerling
}

Theorem 0.1. Suppose that there is no inner model with a Woodin cardinal. Suppose that $\Omega$ is a measurable cardinal. Let $K$ be the Steel core model as computed in $V_{\Omega}$. Let $\kappa \geq \omega_{2}$ and $\lambda=\left(\kappa^{+}\right)^{K}$. Then $\operatorname{cf}(\lambda) \geq \operatorname{card}(\kappa)$.

The main result of [MiSchSt] is that Theorem 0.1 holds under the additional assumption that $\operatorname{card}(\kappa)$ is countably closed. But often, in applications, countable closure is not available. Theorem 0.1 also builds on the earlier covering theorems of Jensen, Dodd and Jensen, and Mitchell; some of the relevant papers are [DeJe], [DoJe1], [DoJe2], [Mi1], [Mi2], and [Je]. The results for smaller core models do not require the existence of a measurable cardinal; it is not known if the large cardinal hypothesis on $\Omega$ can be eliminated completely from Theorem 0.1 (see [Sch2]).

In this paper, we outline a proof of Theorem 0.1. By $K^{c}$, we mean Steel's background certified core model. We shall reduce what we must prove to some iterability properties for $K^{c}$ (labeled "facts" in the proof). In turn, Steel has shown that $K^{c}$ is sufficiently iterable, using the methods in [St, §9]. The proof of Theorem 0.1 is very closely tied to the proof in [MiSchSt], to which we shall refer freely.

\section{An internally approachable chain}

Our proof of Theorem 0.1 begins much as the proof of Jensen's covering theorem for $L$, with an internally approachable chain. Fix $\Omega, \kappa$, and $\lambda$ as in the statement Theorem 0.1, and assume for contradiction that $\operatorname{cf}(\lambda)<$ $\operatorname{card}(\kappa)$. Let $\varepsilon$ be a regular cardinal with $\operatorname{cf}(\lambda)<\varepsilon$ and $\omega_{2} \leq \varepsilon \leq \operatorname{card}(\kappa)$. Though $\varepsilon=\operatorname{cf}(\lambda)^{+}$would do, we prefer to work in slightly more generality.

Let $\left\langle X_{i} \mid i<\varepsilon\right\rangle$ be a continuous chain of elementary substructures of $V_{\Omega+1}$ such that for all $j<\varepsilon,\left\langle X_{i} \mid i \leq j\right\rangle \in X_{j+1}$, and $X_{j} \cap \varepsilon \in \varepsilon$, and $\operatorname{card}\left(X_{j}\right)=\operatorname{card}\left(X_{j} \cap \varepsilon\right)$. Assume also that $\kappa \in X_{0}$. For $i<\varepsilon$, let $\varepsilon_{i}=X_{i} \cap \varepsilon$. Note that $\left\langle\varepsilon_{i} \mid i<\varepsilon\right\rangle$ is a normal sequence converging to $\varepsilon$.

Received June 26,1995.

The research of the first author was partially supported by NSF Grant DMS-9306286.

The research of the second author was partially supported by an NSF Postdoctoral Fellowship. 
For $i<\varepsilon$, let $\pi_{i}: N_{i} \longrightarrow V_{\Omega+1}$ be the uncollapse of $X_{i}$. So $\operatorname{crit}\left(\pi_{i}\right)=\varepsilon_{i}$. We call a partial function $F$ on $\varepsilon$ a choice function if and only if $F(i) \in X_{i}$ for all $i \in \operatorname{dom}(F)$.

Lemma 1.1. Suppose that $F$ is a choice function and that $\operatorname{dom}(F)$ is stationary in $\varepsilon$. Then there is a stationary $S \subseteq \operatorname{dom}(F)$ on which $F$ is constant. Moreover, if this constant value is an ordinal $\geq \varepsilon$, then the map $i \mapsto\left(\pi_{i}\right)^{-1}(F(i))$ is strictly increasing on $S$.

Proof. Let $\left\langle G_{i} \mid i<\varepsilon\right\rangle$ be a sequence, strictly increasing and continuous with respect to inclusion, such that for all $i<\varepsilon, G_{i}$ is a function from $\varepsilon_{i}$ onto $X_{i}$. Let $C=\left\{i<\varepsilon \mid \varepsilon_{i}=i\right\}$. Then $C$ is club and if $i \in C$, then $\operatorname{crit}\left(\pi_{i}\right)=\varepsilon_{i}=i$. Define $H$ on $\operatorname{dom}(F) \cap C$ by $H(i)=\left(G_{i}\right)^{-1}(F(i))$. Then $H(i)<\varepsilon_{i}=i$ for all $i \in \operatorname{dom}(H)$. By Fodor's lemma, there is a stationary set $S \subseteq \operatorname{dom}(H)$ on which $H$ is constant. Suppose that $i, j \in S$ and $i<j$. Then $F(i)=G_{i}(H(i))=G_{j}(H(i))=G_{j}(H(j))=F(j)$. Therefore, $F$ is constant on $S$.

Suppose that $F$ maps into the ordinals. It is clear that $i \mapsto\left(\pi_{i}\right)^{-1}(F(i))$ is nondecreasing on $S$. Suppose that $i<j$ are both in $S$ and that $F(i)=$ $F(j) \geq \varepsilon$. Then $\left(\pi_{i}\right)^{-1}(F(i))<\mathrm{OR}^{N_{i}}<\operatorname{crit}\left(\pi_{j}\right)=\varepsilon_{j}=\left(\pi_{j}\right)^{-1}(\varepsilon) \leq$ $\left(\pi_{j}\right)^{-1}(F(j))$.

Notation 1.2. Suppose that $\mathcal{T}$ is an iteration tree. We shall write $\mathcal{M}(\mathcal{T}, \eta)$ for $\mathcal{M}_{\eta}^{\mathcal{T}}$ and $E(\mathcal{T}, \eta)$ for $E_{\eta}^{\mathcal{T}}$. If $\mathcal{T}$ has successor length, then we denote the last model of $\mathcal{T}$ by $\mathcal{M}_{\infty}(\mathcal{T})$.

Notation 1.3. Suppose that $N$ and $M$ are transitive and $\pi: N \longrightarrow M$ is sufficiently elementary. Suppose that $\bar{\kappa}<\mathrm{OR}^{N}$. Let $E$ be the long extender of length $\pi(\bar{\kappa})$ derived from $\pi$. Suppose that $\mathcal{P}$ is a premouse with $\bar{\kappa}<\mathrm{OR}^{\mathcal{P}}$ and that $E$ is a long extender over $\mathcal{P}$. If $\mathcal{P}$ is a set premouse and, for some $n<\omega, \rho_{n+1}^{\mathcal{P}} \leq \bar{\kappa}$, then we set $n(\mathcal{P}, \bar{\kappa})$ equal to the least such $n$. Also, if $n=n(\mathcal{P}, \bar{\kappa})$, then we write $\operatorname{ult}(\mathcal{P}, \pi, \bar{\kappa})$ for $\operatorname{ult}_{n}(\mathcal{P}, E)$. If, on the other hand, $\mathcal{P}$ is a weasel, then we write $\operatorname{ult}(\mathcal{P}, \pi, \bar{\kappa})$ for $\operatorname{ult}_{0}(\mathcal{P}, E)$.

Fix an inaccessible cardinal $\Gamma<\Omega$ such that $\Gamma>\lambda$. Let $W$ be the canonical very soundness witness for $\mathcal{J}_{\Gamma}^{K}$. We assume that $\Gamma \in X_{0}$.

In [MiSchSt], a single hull $X \prec V_{\Omega+1}$ was considered; $N$ was the transitive collapse of $X$, and various objects related to the coiteration of $\left(W^{N}, W\right)$ were identified. Here we have a chain of $\varepsilon$-many hulls $X_{i}$. We shall use a subscript or a superscript $i$ on the name of the object identified in [MiSchSt] to indicate that it corresponds to the hull $X_{i}$.

Notation 1.4 .

(a) Let $W^{i}=W^{N_{i}}$. 
(b) Let $\left(\overline{\mathcal{T}}^{i}, \mathcal{T}^{i}\right)$ be the pair of iteration trees resulting from the coiteration of $\left(W^{i}, W\right)$.

(c) Let $\theta^{i}+1$ be the common length of $\mathcal{T}^{i}$ and $\overline{\mathcal{T}}^{i}$.

(d) Let $\Gamma^{i}=\left(\pi_{i}\right)^{-1}(\Gamma)$.

(e) Let $\vec{\kappa}^{i}=\left\langle\kappa_{\alpha}^{i} \mid \alpha \leq \gamma^{i}\right\rangle$ be the increasing list of cardinals of $\mathcal{M}_{\infty}\left(\overline{\mathcal{T}}^{i}\right)$ up to and including $\Gamma^{i}$. That is, $\vec{\kappa}^{i}$ is the initial segment of the $\aleph$-function up to and including $\Gamma^{i}$ in the last model of $\overline{\mathcal{T}}^{i}$. (In fact, $\gamma^{i}=\Gamma^{i}$.)

(f) For $\alpha \leq \gamma^{i}$, let $\lambda_{\alpha}^{i}$ be the successor cardinal of $\kappa_{\alpha}^{i}$ in $\mathcal{M}_{\infty}\left(\overline{\mathcal{T}}^{i}\right)$. So $\lambda_{\alpha}^{i}=\kappa_{\alpha+1}^{i}$ whenever $\alpha<\gamma^{i}$. Put $\vec{\lambda}^{i}=\left\langle\lambda_{\alpha}^{i} \mid \alpha<\gamma^{i}\right\rangle$ (the sequence of length $\gamma^{i}$ ).

(g) For $\alpha \leq \gamma^{i}$, let $\eta^{i}(\alpha)$ be the least $\eta<\theta^{i}$ such that $E\left(\mathcal{T}^{i}, \eta\right)$ has generators $\geq \kappa_{\alpha}^{i}$, if such an $\eta$ exists, and put $\eta^{i}(\alpha)=\theta^{i}$ if no such $\eta$ exists.

(h) Let $\left(\overrightarrow{\mathcal{P}}^{i}, \vec{\lambda}^{i}\right)$ be the phalanx of length $\gamma^{i}+1$ derived from $\mathcal{T}^{i}$. This means that for every $\alpha \leq \gamma^{i}, \mathcal{P}_{\alpha}^{i}$ is the longest initial segment of $\mathcal{M}\left(\mathcal{T}^{i}, \eta^{i}(\alpha)\right)$ with just the subsets of $\kappa_{\alpha}^{i}$ constructed before $\lambda_{\alpha}^{i}$. $\mathcal{P}_{\alpha}^{i}$ might be a set premouse, or it might be a weasel; we cannot rule out either case.

(i) For $\alpha<\gamma^{i}$, let $\mathcal{R}_{\alpha}^{i}=\operatorname{ult}\left(\mathcal{P}_{\alpha}^{i}, \pi_{i}, \kappa_{\alpha}^{i}\right)$. This definition assumes that the $\left(\varepsilon_{i}, \pi_{i}\left(\kappa_{\alpha}^{i}\right)\right)$ long extender derived from $\pi_{i}$ measures sets in $\mathcal{P}_{\alpha}^{i}$ (which would follow from hypothesis $(1)_{\alpha}^{i}$ of Definition 1.5 below). We allow for the possibility that $\mathcal{R}_{\alpha}^{i}$ is ill-founded. Even if $\mathcal{R}_{\alpha}^{i}$ is well-founded, it seems possible that $\mathcal{R}_{\alpha}^{i}$ is not a potential premouse (ppm), as $\dot{F}^{\mathcal{R}_{\alpha}^{i}}$, the last predicate of $\mathcal{R}_{\alpha}^{i}$, might code an extender fragment, rather than a total extender, over $\mathcal{R}_{\alpha}^{i}$.

(j) Let $\pi_{\alpha}^{i}: \mathcal{P}_{\alpha}^{i} \longrightarrow \mathcal{R}_{\alpha}^{i}$ be the ultrapower map.

(k) In [MiSchSt], a premouse $\mathcal{S}_{\alpha}^{i}$ is defined from $\mathcal{T}^{i} \uparrow\left(\eta^{i}(\alpha)+1\right)$ and $\pi_{i}$. When $\mathcal{R}_{\alpha}^{i}$ is a premouse, then $\mathcal{S}_{\alpha}^{i}=\mathcal{R}_{\alpha}^{i}$; but otherwise, $\mathcal{S}_{\alpha}^{i} \neq$ $\mathcal{R}_{\alpha}^{i}$. $\mathcal{S}_{\alpha}^{i}$ substitutes for $\mathcal{R}_{\alpha}^{i}$ in many roles. The most important difference is that $\mathcal{S}_{\alpha}^{i}$ is a premouse, even if $\mathcal{R}_{\alpha}^{i}$ is not a premouse.

(l) Let $\mathcal{Q}_{\alpha}^{i}$ be the structure defined from $\mathcal{P}_{\alpha}^{i}$ by analogy with how $\mathcal{S}_{\alpha}^{i}$ was defined from $\mathcal{R}_{\alpha}^{i}$. In fact, $\mathcal{S}_{\alpha}^{i}=\operatorname{ult}\left(\mathcal{Q}_{\alpha}^{i}, \pi_{i}, \kappa_{\alpha}^{i}\right)$.

(m) Let $\Lambda_{\alpha}^{i}=\sup \left(\pi_{i}{ }^{\prime \prime} \lambda_{\alpha}^{i}\right)$. Then $\Lambda_{\alpha}^{i}=\left(\pi_{i}\left(\kappa_{\alpha}^{i}\right)^{+}\right)^{\mathcal{S}_{\alpha}^{i}}$.

Definition 1.5. For each $i<\varepsilon$ and $\alpha<\gamma^{i}$, we name the following six properties:

$(1)_{\alpha}^{i} \quad$ if $\eta \leq \theta^{i}$ and $E\left(\overline{\mathcal{T}}^{i}, \eta\right) \neq \emptyset$, then $\operatorname{lh}\left(E\left(\overline{\mathcal{T}}^{i}, \eta\right)\right)>\lambda_{\alpha}^{i}$;

$(2)_{\alpha}^{i} \quad\left(\left(W, \mathcal{S}_{\alpha}^{i}\right), \pi_{\alpha}^{i}\left(\kappa_{\alpha}^{i}\right)\right)$ is an iterable phalanx;

$(3)_{\alpha}^{i} \quad\left(\left(W^{i}, \mathcal{Q}_{\alpha}^{i}\right), \kappa_{\alpha}^{i}\right)$ is an iterable phalanx; 
$(4)_{\alpha}^{i} \quad\left(\left(\overrightarrow{\mathcal{P}}^{i}\left\lceil\alpha, W^{i}\right), \vec{\lambda}^{i} \uparrow \alpha\right)\right.$ is an iterable phalanx;

$(5)_{\alpha}^{i} \quad\left(\left(\overrightarrow{\mathcal{R}}^{i} \uparrow \alpha, W\right), \vec{\Lambda}^{i} \uparrow \alpha\right)$ is an iterable phalanx;

$(6)_{\alpha}^{i} \quad\left(\left(\overrightarrow{\mathcal{S}}^{i} \uparrow \alpha, W\right), \vec{\Lambda}^{i} \uparrow \alpha\right)$ is an iterable phalanx.

Lemma 1.6. Consider any $i<\varepsilon$.

(a) If $(1)_{\alpha}^{i}-(6)_{\alpha}^{i}$ hold for every $\alpha<\gamma^{i}$, then Theorem 0.1 holds.

(b) If $\pi_{i}$ is continuous at ordinals of countable cofinality, then the following implications hold for any $\alpha<\gamma^{i}$.

$$
\begin{gathered}
(6)_{\alpha}^{i} \Longrightarrow(5)_{\alpha}^{i} \Longrightarrow(4)_{\alpha}^{i} \Longrightarrow(1)_{\alpha}^{i} \\
\forall \beta<\alpha(4)_{\beta}^{i} \Longrightarrow(3)_{\alpha}^{i} \\
\forall \beta<\alpha(2)_{\beta}^{i} \Longrightarrow(6)_{\alpha}^{i}
\end{gathered}
$$

Lemma 1.6 was proved in [MiSchSt], where it was also argued that if ${ }^{\omega} X_{i} \subset X_{i}$, then $(3)_{\alpha}^{i} \Longrightarrow(2)_{\alpha}^{i}$ for every $\alpha<\gamma^{i}$, and consequently, Theorem 0.1 holds. We shall show that a weaker closure condition on $X_{i}$ suffices, and holds for a stationary set of $i<\varepsilon$. In light of Lemma 1.6(a) and our denial of Theorem 0.1, we may make the following definition.

Definition 1.7. For any $i<\varepsilon$, define $\alpha^{i}$ to be the least $\alpha$ such that at least one of $(1)_{\alpha}^{i}-(6)_{\alpha}^{i}$ fails.

If $\operatorname{cf}(i)>\omega$, then $\pi_{i}$ is continuous at ordinals of countable cofinality, and so Lemma 1.6 implies that $(1)_{\alpha^{i}}^{i}$ and $(3)_{\alpha^{i}}^{i}-(6)_{\alpha^{i}}^{i}$ hold, while $(2)_{\alpha^{i}}^{i}$ fails. We shall use the following notation:

$$
\begin{aligned}
& \kappa^{i}=\kappa_{\alpha^{i}}^{i} \quad \eta^{i}=\eta^{i}\left(\alpha^{i}\right) \quad \mathcal{P}^{i}=\mathcal{P}_{\alpha^{i}}^{i} \quad \Lambda^{i}=\Lambda_{\alpha^{i}}^{i} \quad \mathcal{R}^{i}=\mathcal{R}_{\alpha^{i}}^{i} \\
& \lambda^{i}=\lambda_{\alpha^{i}}^{i} \quad \mathcal{Q}^{i}=\mathcal{Q}_{\alpha^{i}}^{i} \quad \mathcal{S}^{i}=\mathcal{S}_{\alpha^{i}}^{i}
\end{aligned}
$$

From now on, we shall write $\overrightarrow{\mathcal{P}}^{i}$ when we mean $\overrightarrow{\mathcal{P}}^{i} \uparrow \alpha^{i}$. As we shall never again refer to coordinates of $\overrightarrow{\mathcal{P}}^{i}$ beyond $\alpha^{i}$, there is no ambiguity. The same goes for $\overrightarrow{\mathcal{Q}}^{i}, \vec{\kappa}^{i}, \vec{\lambda}^{i}, \overrightarrow{\mathcal{R}}^{i}, \overrightarrow{\mathcal{S}}^{i}$, and $\vec{\Lambda}^{i}$.

By Lemma 1.1, there is a stationary set $S \subseteq\left\{i<\varepsilon \mid \operatorname{cf}(i)>\omega \wedge \varepsilon_{i}=i\right\}$ on which the following choice functions are constant:

$$
\begin{aligned}
i \mapsto \pi_{i}\left(\alpha^{i}\right) \quad i & \mapsto \pi_{i}\left(\kappa^{i}\right) \quad i \mapsto \pi_{i}\left(\eta^{i}\right) & i \mapsto n\left(\mathcal{P}^{i}, \kappa^{i}\right) \\
& i \mapsto \pi_{i}\left(\lambda^{i}\right) & i \mapsto n\left(\mathcal{Q}^{i}, \kappa^{i}\right)
\end{aligned}
$$

Then $i \mapsto \alpha^{i}$ and $i \mapsto \eta^{i}$ are non-decreasing on $S$, while $i \mapsto \kappa^{i}$ and $i \mapsto \lambda^{i}$ are strictly increasing on $S$. (Note that $\kappa^{i} \geq \varepsilon_{i}$, since $\left((W, W), \varepsilon_{i}\right)$ is iterable; hence $\pi_{i}\left(\kappa^{i}\right) \geq \varepsilon$. Apply Lemma 1.1.) 


\section{A pull-back $\mathcal{Q}^{*}$ of $\mathcal{Q}^{j}$}

Let $S$ be the stationary set from $\S 1$. For the rest of this paper, fix $j \in S \cap \lim (S)$. Since $j \in S,(2)_{\alpha^{j}}^{j}$ fails. Let $\mathcal{U}$ be an ill behaved iteration tree on $\left(\left(W, \mathcal{S}^{j}\right), \pi_{j}\left(\kappa^{j}\right)\right)$. We include here the possibility that $\mathcal{S}^{j}$ itself is ill-founded, which would mean that $\mathcal{U}$ is the empty tree.

Let $\psi: M \longrightarrow V_{\Omega+1}$ be elementary with $\mathrm{M}$ countable and transitive with everything relevant in the range of $\psi$. Say $\mathcal{U}=\psi\left(\mathcal{U}^{\prime}\right), W=\psi\left(W^{\prime}\right)$, $\mathcal{S}^{j}=\psi\left(\mathcal{S}^{\prime}\right)$, and $\pi_{j}\left(\kappa^{j}\right)=\psi\left(\kappa^{\prime}\right)$. $\mathcal{U}^{\prime}$ is a countable, ill behaved iteration tree on $\left(\left(W^{\prime}, \mathcal{S}^{\prime}\right), \kappa^{\prime}\right)$, and $\psi^{\prime \prime}\left(\mathcal{U}^{\prime}\right)$, the copy of $\mathcal{U}$ by $\psi$ is a countable, ill behaved iteration tree on $\left(\left(W, \mathcal{S}^{j}\right), \pi_{j}\left(\kappa^{j}\right)\right)$.

We remark that in [MiSchSt, 3.13], the countable completeness of the extender $E_{\pi}$ derived from $\pi$ was used to find maps from $\left(\left(W^{\prime}, \mathcal{S}^{\prime}\right), \kappa^{\prime}\right)$ into $\left(\left(W^{j}, \mathcal{Q}^{j}\right), \kappa^{j}\right)$. These maps were then used to copy $\mathcal{U}^{\prime}$ to an ill behaved iteration tree on $\left(\left(W^{j}, \mathcal{Q}^{j}\right), \kappa^{j}\right)$, thereby contradicting $(3)_{\alpha^{j}}^{j}$. But here, $E_{\pi}$ is not countably complete.

For the rest of this paper, fix $i \in S$ such that $i<j$ and $\operatorname{ran}(\psi) \cap X_{j} \subset X_{i}$. This is possible since $j \in \lim (S)$ and $\operatorname{cf}(j)>\omega$. Let $\pi_{i, j}: N_{i} \longrightarrow N_{j}$ be the natural embedding, that is, the uncollapse of $\left(\pi_{j}\right)^{-1}\left(X_{i}\right)$. We have the following commutative diagram.

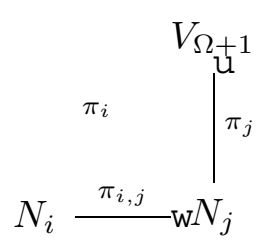

By a standard fine structural construction, we shall define a "pull-back" of $\mathcal{Q}^{j}$ to a premouse $\mathcal{Q}^{*}$ that agrees with $\mathcal{Q}^{i}$ below $\lambda^{i}$. This is done in two cases, depending on whether or not $\mathcal{Q}^{j}$ is a proper class. In both cases, $\mathcal{Q}^{*}$ ends up being an appropriate hull in $\mathcal{Q}^{j}$ of $\pi_{i, j}$ " $\kappa^{i}$ and a parameter (part of what we need to show is that no new ordinals $<\kappa^{j}$ get into this hull).

Lemma 2.1. Suppose that $\mathcal{Q}^{j}$ is a set premouse. Let $n=n\left(\mathcal{Q}^{j}, \kappa^{j}\right)$. There is a premouse $\mathcal{Q}^{*}$ with the following properties:

(a) $\mathcal{Q}^{*}$ and $W^{i}$ agree below $\lambda^{i}$;

(b) $\lambda^{i}=\left(\kappa_{i}^{+}\right)^{\mathcal{Q}^{*}}$;

(c) $\mathcal{Q}^{*}$ is $\kappa^{i}$-sound;

(d) $n\left(\mathcal{Q}^{*}, \kappa^{i}\right)=n$;

(e) $\mathcal{Q}^{j}=u l t\left(\mathcal{Q}^{*}, \pi_{i, j}, \kappa^{i}\right)$;

(f) the ultrapower map $\pi^{*}: \mathcal{Q}^{*} \longrightarrow \mathcal{Q}^{j}$ is an $n$-embedding such that

$$
\pi^{*}\left\lceil J_{\lambda^{i}}^{\mathcal{Q}^{*}}=\pi_{i, j} \uparrow J_{\lambda^{i}}^{W^{i}} .\right.
$$


Sketch. Recall that $\mathcal{Q}^{j}$ is $\kappa^{j}$-sound in this case, with $\lambda^{j}=\left(\left(\kappa^{j}\right)^{+}\right)^{\mathcal{Q}^{j}}$, and that $\mathcal{Q}^{j}$ and $W^{j}$ agree below $\lambda^{j}$. Also, recall that $\mathcal{S}^{j}=\operatorname{ult}\left(\mathcal{Q}^{j}, \pi_{j}, \kappa^{j}\right)$ is $\pi_{j}\left(\kappa^{j}\right)$-sound, and $\Lambda^{j}=\sup \left(\pi^{\prime \prime} \lambda^{j}\right)$. The following claim implies that $\pi_{i, j}$ is continuous at $\lambda^{i}$; that is, $\lambda^{j}=\pi_{i, j}\left(\lambda^{i}\right)=\sup \left(\pi_{i, j}{ }^{\prime \prime} \lambda^{i}\right)$.

Claim 2.1.1. If $\mathcal{Q}^{j}$ is a set premouse, then the map $\psi: M \longrightarrow V_{\Omega+1}$ is cofinal in $\Lambda^{j}$.

Suppose, to the contrary, that $\operatorname{ran}(\psi) \cap \Lambda^{j}$ is bounded in $\Lambda^{j}$. We can use the condensation theorem, [Sch1, 2.8], to find a proper initial segment $\mathcal{L}$ of $\mathcal{S}^{j}$, an almost $\Sigma_{n+1}$-embedding $\varphi$, and a $\Sigma_{n+1}$-elementary embedding $\widetilde{\psi}$ such that following diagram commutes.

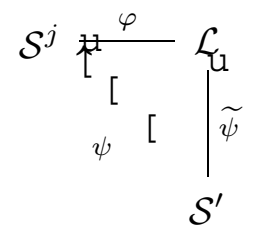

Moreover, we may arrange that $\rho_{n+1}(\mathcal{L})=\pi_{j}\left(\kappa^{j}\right)$ and $\sup \left(\operatorname{ran}(\psi) \cap \Lambda^{j}\right)<$ $\left(\pi_{j}\left(\kappa^{j}\right)^{+}\right)^{\mathcal{L}}=\operatorname{crit}(\varphi)<\Lambda^{j}$. This allows us to use the pair of maps $((\psi \uparrow$ $W), \widetilde{\psi})$ to copy $\mathcal{U}^{\prime}$ to an ill behaved iteration tree on $\left((W, \mathcal{L}), \pi_{j}\left(\kappa^{j}\right)\right)$. Since $\mathcal{S}^{j}$ and $W$ agree below $\Lambda^{j}, \mathcal{L}$ is a proper initial segment of $W$. Since $W$ is iterable, $\left((W, \mathcal{L}), \pi_{j}\left(\kappa^{j}\right)\right)$ is iterable. This contradiction completes the sketch of Claim 2.1.1.

Because $\mathcal{Q}^{j}$ is $\kappa^{j}$-sound, there is a directed system $D \subset J_{\lambda^{j}}^{\mathcal{Q}^{j}}$ such that $\mathcal{Q}^{j}$ is the direct limit of $D$. We take $D$ to have as structures, premice of the form:

$$
\mathcal{H}_{n+1}^{\mathcal{Q}^{j}\lceil\xi}\left(\kappa^{j} \cup p\left(\mathcal{Q}^{j}, \kappa^{j}\right)\right)
$$

for $\xi<\mathrm{OR}^{\mathcal{Q}^{j}}$. The maps of $D$ are the natural $\Sigma_{n}$-elementary maps between the structures of $D$.

Let $D^{*}$ be the directed system whose structures are of the form: $\pi_{i, j}^{-1}(\mathcal{H})$ for some structure $\mathcal{H}$ of $D$ with $\mathcal{H} \in \operatorname{ran}\left(\pi_{i, j}\right)$. Likewise for the maps of $D^{*}$. Then $D^{*} \subset J_{\lambda^{i}}^{\mathcal{Q}^{i}}$. Let $\mathcal{Q}^{*}$ be the direct limit of $D^{*}$, and let $\pi^{*}: \mathcal{Q}^{*} \longrightarrow \mathcal{Q}^{j}$ be the natural map. Clearly, $\pi^{*}$ is $\Sigma_{n}$-elementary. But from Claim 2.1.1, it follows that $\pi^{*}$ is cofinal, and therefore $\Sigma_{n+1}$-elementary. The lemma now follows by standard calculations.

Lemma 2.2. Suppose that $\mathcal{Q}^{j}$ is a weasel. There is a set premouse $\mathcal{Q}^{*}$ such that, if we set $\lambda^{*}=\left(\left(\kappa^{i}\right)^{+}\right)^{\mathcal{Q}^{*}}$, then the following hold:

(a) $\mathcal{Q}^{*}$ and $W^{i}$ agree below $\lambda^{*}$;

(b) $\lambda^{*} \leq \lambda^{i}$; 
(c) there is an elementary embedding $\pi^{*}: \mathcal{Q}^{*} \longrightarrow \mathcal{Q}^{j}$ such that

$$
\pi^{*} \uparrow J_{\lambda^{*}}^{\mathcal{Q}^{*}}=\pi_{i, j} \uparrow J_{\lambda^{*}}^{\mathcal{Q}^{i}}
$$

Sketch. Let $D$ be the directed system consisting of transitive premice of the form $\mathcal{H}_{\omega}^{\mathcal{Q}^{j}}\left(\kappa^{j} \cup\{x\}\right)$ with $x \in \operatorname{ran}(\psi) \cap\left|\mathcal{Q}^{j}\right|$. Pull $D$ back using $\pi_{i, j}$ to a system $D^{*}$. Let $\mathcal{Q}^{*}$ be the direct limit and let $\pi^{*}: \mathcal{Q}^{*} \longrightarrow \mathcal{Q}^{j}$ be the natural elementary embedding.

Lemma 2.3. $\left(\left(W, \operatorname{ult}\left(\mathcal{Q}^{*}, \pi_{i}, \kappa^{i}\right)\right), \pi_{i}\left(\kappa^{i}\right)\right)$ is not iterable.

Proof. We have the following commutative diagram.

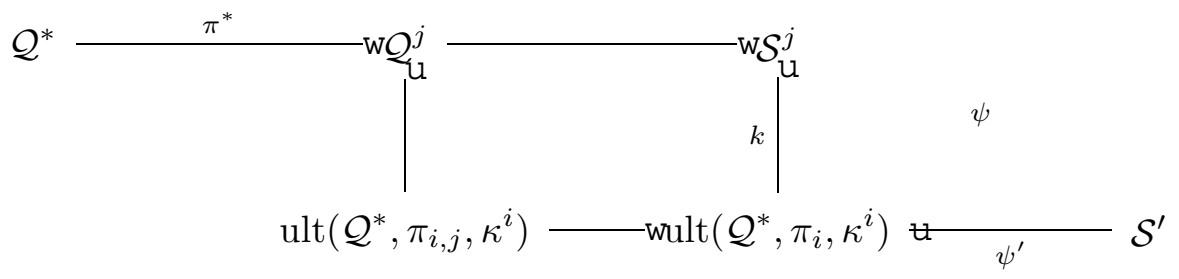

And, $\operatorname{crit}(k)>\pi_{i}\left(\kappa^{i}\right)$. So we can use the pair of embeddings $\left(\psi, \psi^{\prime}\right)$ to copy $\mathcal{U}^{\prime}$ to an ill behaved iteration tree on $\left(\left(W, \operatorname{ult}\left(\mathcal{Q}^{*}, \pi_{i}, \kappa^{i}\right)\right), \pi_{i}\left(\kappa^{i}\right)\right)$

It is worth noting that the map from $\operatorname{ult}\left(\mathcal{Q}^{*}, \pi_{i, j}, \kappa^{i}\right)$ into $\mathcal{Q}^{j}$ in the diagram above is elementary and has critical point strictly greater than $\kappa^{j}$. In fact, if $\mathcal{Q}^{j}$ is a set premouse, then the map is the identity.

\section{Definition 2.4.}

(a) A premouse $\mathcal{M}$ is $\infty$-bad iff $\left(\left(W, \operatorname{ult}\left(\mathcal{M}, \pi_{i}, \kappa^{i}\right)\right), \pi_{i}\left(\kappa^{i}\right)\right)$ is a phalanx, but is not iterable.

(b) $\mathcal{M}$ is $j$-bad iff $\left(\left(W^{j}, \operatorname{ult}\left(\mathcal{M}, \pi_{i, j}, \kappa^{i}\right)\right), \kappa^{j}\right)$ is a phalanx, but is not iterable.

\section{Corollary 2.5.}

(a) $\mathcal{Q}^{i}$ is $\infty$-bad.

(b) $\mathcal{Q}^{*}$ is $\infty$-bad.

(c) $\mathcal{Q}^{*}$ is not $j$-bad.

Proof. By our choice of $\alpha^{i},(2)_{\alpha^{i}}^{i}$ fails. Therefore, clause (a) holds. Clause (b) follows from Lemma 2.3. Recall that $(3)_{\alpha^{j}}^{j}$ holds and asserts that $\left(\left(W^{j}, \mathcal{Q}^{j}\right), \kappa^{j}\right)$ is an iterable phalanx. Since $\operatorname{ult}\left(\mathcal{Q}^{*}, \pi_{i, j}, \kappa^{i}\right)$ embeds into $\mathcal{Q}^{j}$ with critical point greater than $\kappa^{j}$, clause (c) holds.

Lemma 2.6. If $\mathcal{M}$ is $\infty$-bad and $\mathcal{M} \in \operatorname{ran}\left(\pi_{j}\right)$, then $\mathcal{M}$ is $j$-bad. In particular, $\mathcal{Q}^{i}$ is $j$-bad. 
Proof. Since $\pi_{j}$ is elementary and $\pi_{j}^{-1}\left(\pi_{i}\left\lceil\pi_{i}\left(\kappa^{i}\right)\right)=\pi_{i, j} \uparrow \kappa^{j}\right.$, we have that

$$
N_{j} \vDash "\left(\left(W^{j}, \operatorname{ult}\left(\pi_{j}^{-1}(\mathcal{M}), \pi_{i, j}, \kappa^{i}\right)\right), \kappa^{j}\right) \text { is not iterable." }
$$

By absoluteness (using the generic branch formulation of iterability),

$$
\left(\left(W^{j}, \operatorname{ult}\left(\pi_{j}^{-1}(\mathcal{M}), \pi_{i, j}, \kappa^{i}\right)\right), \kappa^{j}\right)
$$

is not iterable. By the shift lemma, we have a map $k$ with $\operatorname{crit}(k) \geq \kappa^{j}$ so that the following diagram commutes:

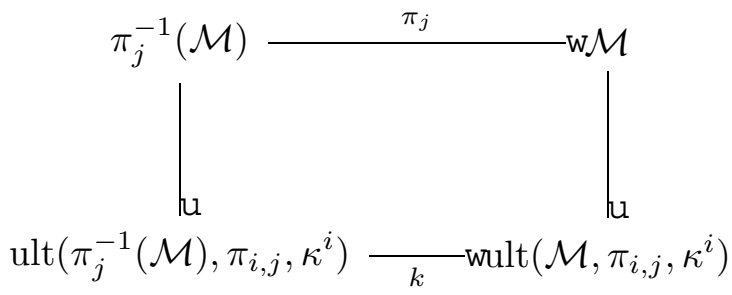

An ill behaved iteration tree on $\left(\left(W^{j}, \operatorname{ult}\left(\pi_{j}^{-1}(\mathcal{M}), \pi_{i, j}, \kappa^{i}\right)\right), \kappa^{j}\right)$ can be copied to an ill behaved iteration tree on $\left(\left(W^{j}, \operatorname{ult}\left(\mathcal{M}, \pi_{i, j}, \kappa^{i}\right)\right), \kappa^{j}\right)$ using the pair ((id $\left.\left.\uparrow W^{j}\right), k\right)$. So $\mathcal{M}$ is $j$-bad.

In light of Corollary 2.5(c) and Lemma 2.6, we would have a contradiction if we could show that $\mathcal{Q}^{i}$ embeds into $\mathcal{Q}^{*}$ with critical point at least $\kappa^{i}$. This is a first approximation to our general strategy.

Definition 2.7. A premouse $\mathcal{M}$ is $i$-good iff $\left(\left(\overrightarrow{\mathcal{P}}^{i}, \mathcal{M}\right), \vec{\lambda}^{i}\right)$ is an iterable phalanx.

Fact 2.8. (Steel). $\mathcal{Q}^{i}$ is i-good.

The fact is proved using the methods of [St, §9]. Much of the rest of this section will be taken up with showing that $\mathcal{Q}^{*}$ is also $i$-good.

Definition 2.9. Let $\Lambda_{\beta}^{i, j}=\sup \left(\pi_{i, j}{ }^{i} \lambda^{i}\right), \mathcal{R}_{\beta}^{i, j}=\operatorname{ult}\left(\mathcal{P}^{i}, \pi_{i, j}, \kappa_{\beta}^{i}\right)$, and $\mathcal{S}_{\beta}^{i, j}=\operatorname{ult}\left(\mathcal{Q}_{\beta}^{i}, \pi_{i, j}, \kappa_{\beta}^{i}\right)$, for any $\beta<\alpha^{i}$.

Lemma 2.10. Let $\beta<\alpha^{i}$. There is an iteration tree $\mathcal{V}_{\beta}$ on $W$ such that

(a) $\mathcal{V}_{\beta}$ extends $\mathcal{T}^{j} \uparrow\left(\eta^{j}\left(\pi_{i, j}(\beta)\right)+1\right)$;

(b) $\mathcal{V}_{\beta}$ has a last model;

(c) there is $\mathcal{N}_{\beta}$, a premouse, and $\varphi_{\beta}: \mathcal{S}_{\beta}^{i, j} \longrightarrow \mathcal{N}_{\beta}$, an elementary embedding, such that $\mathcal{N}_{\beta}$ is an initial segment of $\mathcal{M}_{\infty}\left(\mathcal{V}_{\beta}\right)$, and $\operatorname{crit}\left(\varphi_{\beta}\right) \geq \pi_{i, j}\left(\kappa_{\beta}^{i}\right)$. 
Sketch. Fix $\beta<\alpha^{i}$. Intuitively, the idea is to compare $\mathcal{S}^{i, j}$ and $\mathcal{T}^{j}$. Suppose that

$$
\left(\left(\overrightarrow{\mathcal{P}}^{j}\left\lceil\pi_{i, j}(\beta), \mathcal{S}_{\beta}^{i, j}\right), \vec{\lambda}^{j}\left\lceil\pi_{i, j}(\beta)\right)\right.\right.
$$

and

$$
\left(\left(\overrightarrow{\mathcal{P}}^{j}\left\lceil\pi_{i, j}(\beta), \mathcal{P}_{\pi_{i, j}(\beta)}^{j}\right), \vec{\lambda}^{j}\left\lceil\pi_{i, j}(\beta)\right)\right.\right.
$$

are coiterable, and that $(\mathcal{U}, \mathcal{V})$ is the pair of iteration trees resulting from the coiteration. Then, by standard arguments, the iteration tree $\mathcal{V}$ can be rearranged as the iteration tree $\mathcal{V}_{\beta}$ that we are looking for, with the embedding along the branch from $\mathcal{S}_{\beta}^{i, j}$ to $\mathcal{M}_{\infty}(\mathcal{U})$ serving as $\varphi_{\beta}$. The details are like those in the proof of [MiSchSt, 3.14 and 3.15] (the lemmas that derive $(1)_{\alpha}^{j}$ from $(4)_{\alpha}^{j}$, for $\left.\alpha=\pi_{i, j}(\beta)\right)$. The second phalanx displayed above is iterable, since $W$ is. The first phalanx is also iterable, as we now argue.

By a standard copying argument, it is enough to show that the phalanx

$$
\left(\left(\overrightarrow{\mathcal{R}}^{j} \uparrow \pi_{i, j}(\beta), \mathcal{S}_{\beta}^{i}\right), \vec{\Lambda}^{j} \uparrow \pi_{i, j}(\beta)\right)
$$

is iterable. Briefly, for each $\gamma<\pi_{i, j}(\beta)$, we can copy using the ultrapower map $\pi_{\gamma}^{j}: \mathcal{P}_{\gamma}^{j} \longrightarrow \mathcal{R}_{\gamma}^{j}=\operatorname{ult}\left(\mathcal{P}_{\gamma}^{j}, \pi_{j}, \kappa_{\gamma}^{j}\right)$ on $\mathcal{P}_{\gamma}^{j}$. And, we use the map from the diagram

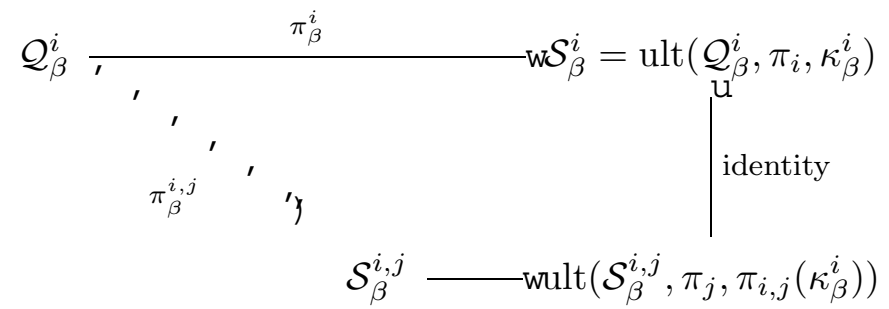

between the starting models $\mathcal{S}_{\beta}^{i, j}$ and $\mathcal{S}_{\beta}^{i}$. All the copying maps agree with $\pi_{j}$ out to the appropriate ordinals.

Next we indicate why it is enough to show that

$$
\left(\left(\overrightarrow{\mathcal{S}}^{j}\left\lceil\pi_{i, j}(\beta), \mathcal{S}_{\beta}^{i}\right), \vec{\Lambda}^{j}\left\lceil\pi_{i, j}(\beta)\right)\right.\right.
$$

is iterable. Recall [MiSchSt, 3.18], the lemma that says $(6)_{\alpha}^{j} \Longrightarrow(5)_{\alpha}^{j}$ whenever $\alpha<\alpha^{j}$, in particular, when $\alpha=\pi_{i, j}(\beta)$. The proof involved a kind of enlargement that differed from the usual copying construction, that used the details of how each $\mathcal{S}_{\gamma}^{j}$ was obtained from $\mathcal{R}_{\gamma}^{j}$. It might be helpful to recall that the enlarged iteration tree had a different tree structure from the given iteration tree. Without giving the details, if we carry out 
the analogous enlargement construction here, we see how to reduce the iterability of $\left(\left(\overrightarrow{\mathcal{R}}^{j}\left\lceil\pi_{i, j}(\beta), \mathcal{S}_{\beta}^{i}\right), \vec{\Lambda}^{j}\left\lceil\pi_{i, j}(\beta)\right)\right.\right.$ to that of

$$
\left(\left(\overrightarrow{\mathcal{S}}^{j} \uparrow \pi_{i, j}(\beta), \mathcal{S}_{\beta}^{i}\right), \vec{\Lambda}^{j}\left\lceil\pi_{i, j}(\beta)\right) .\right.
$$

Now, we outline how to reduce the iterability of the last phalanx to that of a $W$-based phalanx. First, because $(1)_{\beta}^{i}$ and $(2)_{\beta}^{i}$ hold, [MiSchSt, 3.12] gives an iteration tree $\mathcal{Y}$ on $W$ such that $\mathcal{Y}$ has a successor length, and all extenders used on $\mathcal{Y}$ have length at least $\Lambda_{\beta}^{i}$, and the corollary also gives an elementary embedding $k$ from $\mathcal{S}_{\beta}^{i}$ into an initial segment $\mathcal{A}$ of $\mathcal{M}_{\infty}(\mathcal{Y})$, with $\operatorname{crit}(k) \geq \pi_{i}\left(\kappa_{\beta}^{i}\right)$. Similarly, for each $\gamma<\pi_{i, j}(\beta)$, because $(1)_{\gamma}^{j}$ and $(2)_{\gamma}^{j}$ hold, [MiSchSt, 3.12] gives an iteration tree $\mathcal{Y}_{\gamma}$ on $W$ such that $\mathcal{Y}_{\gamma}$ has a last model, and all extenders used on $\mathcal{Y}_{\gamma}$ have length at least $\Lambda_{\gamma}^{j}$, and the corollary also gives an elementary embedding $k_{\gamma}$ from $\mathcal{S}_{\gamma}^{j}$ into an initial segment $\mathcal{A}_{\gamma}$ of $\mathcal{M}_{\infty}\left(\mathcal{Y}_{\gamma}\right)$, with $\operatorname{crit}\left(k_{\gamma}\right) \geq \pi_{j}\left(\kappa_{\gamma}^{j}\right)$. Using the sequence of maps $\left(\left\langle k_{\gamma} \mid \gamma<\pi_{i, j}(\beta)\right\rangle\right.$, $k$ ) we can copy a putative iteration tree on

$$
\left(\left(\overrightarrow{\mathcal{S}}^{j} \uparrow \pi_{i, j}(\beta), \mathcal{S}_{\beta}^{i}\right), \vec{\Lambda}^{j}\left\lceil\pi_{i, j}(\beta)\right)\right.
$$

to an iteration tree on $\left.\left(\left\langle\mathcal{A}_{\gamma} \mid \gamma<\pi_{i, j}(\beta)\right\rangle, \mathcal{A}\right), \vec{\Lambda}^{j} \uparrow \pi_{i, j}(\beta)\right)$. This last phalanx is $W$-based, and therefore iterable, by the main result in [St, $\S 9]$.

There is a small subtlety in the last copying argument, since we must allow for the possibility that $\operatorname{crit}\left(k_{\beta}\right)=\pi_{i}\left(\kappa_{\beta}^{i}\right)$. It is the variation of the usual copying procedure, as explained in [St, §6], and also in the proof of [MiSchSt, 3.16] (deriving $(3)_{\alpha}^{j}$ from $\left.(4)_{\alpha}^{j}\right)$, that we have in mind.

Lemma 2.11. $\mathcal{Q}^{*}$ is $i$-good.

Sketch. We must see that the phalanx $\left(\left(\overrightarrow{\mathcal{P}}^{i}, \mathcal{Q}^{*}\right), \vec{\lambda}^{i}\right)$ is iterable. By the usual copying construction, it is enough to show that $\left(\left(\overrightarrow{\mathcal{R}}^{i, j}, \mathcal{Q}^{j}\right), \overrightarrow{\Lambda^{i, j}}\right)$ is iterable.

We remark that $\overrightarrow{\mathcal{S}}^{i, j}$ is obtained from $\overrightarrow{\mathcal{R}}^{i, j}$ as $\overrightarrow{\mathcal{Q}}^{i}$ was obtained from $\overrightarrow{\mathcal{P}}^{i}$. The proof is like that of the claim in the proof of [MiSchSt, 3.13].

Now recall the proof that $(6)_{\beta}^{i} \Longrightarrow(5)_{\beta}^{i}$ for $\beta<\alpha^{i}$, that is, the proof of [MiSchSt, 3.18]. Using an enlargement similar to the one introduced there, we see that it is enough to show that $\left(\left(\overrightarrow{\mathcal{S}}^{i, j}, \mathcal{Q}^{j}\right), \vec{\Lambda}^{i, j}\right)$ is iterable.

For $\beta<\alpha^{i}$, let $\varphi_{\beta}: \mathcal{S}_{\beta}^{i, j} \longrightarrow \mathcal{N}_{\beta}$ be the map from Lemma 2.10. Then copying using $\left(\left\langle\varphi_{\beta} \mid \beta<\alpha^{i}\right\rangle\right.$, id $\left.\uparrow\left|\mathcal{Q}^{j}\right|\right)$ can be used to see that it is enough to show that

$$
\left(\left(\left\langle\mathcal{N}_{\beta} \mid \beta<\alpha^{i}\right\rangle, \mathcal{Q}^{j}\right), \vec{\Lambda}^{i, j}\right)
$$

is iterable. The following picture illustrates the situation. 


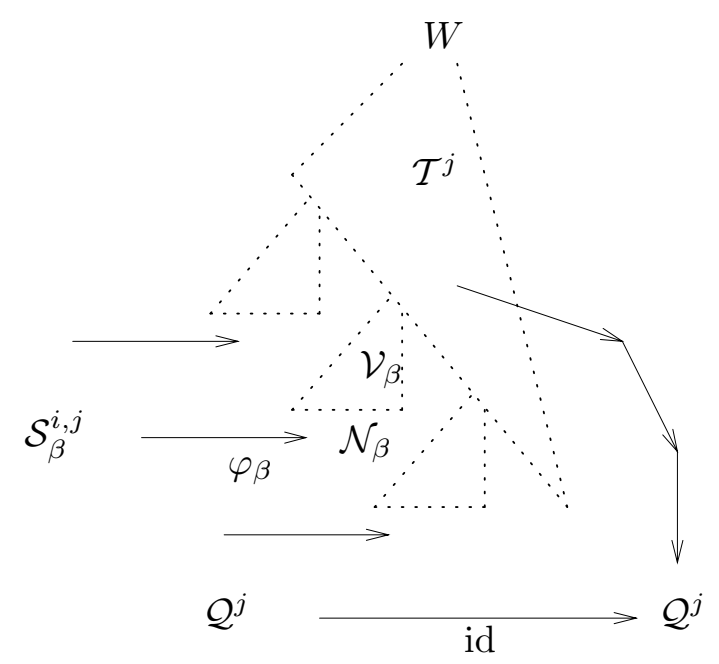

In the last copying construction, we must allow for the possibility that $\operatorname{crit}\left(\varphi_{\beta}\right)=\pi_{i, j}\left(\kappa_{\beta}^{i}\right)$. It is the variation of the usual copying procedure, as explained in [St, $\S 6]$, and also in the proof of [MiSchSt, 3.16], that we have in mind.

Fact 2.11.1 (Steel). $\left(\left(\left\langle\mathcal{N}_{\beta} \mid \beta<\alpha^{i}\right\rangle, \mathcal{Q}^{j}\right), \vec{\Lambda}^{i, j}\right)$ is iterable.

The fact is proved by the methods of [St, §9], and the lemma follows.

Recall that $\mathcal{Q}^{*}$ is not $j$-bad; this was Lemma $2.3(\mathrm{c})$. The following lemma is a strengthening of this fact. It might be read as saying that $\mathcal{Q}^{*}$ is "hereditarily not $j$-bad".

Lemma 2.12. Suppose that $\mathcal{I}$ is an iteration tree on $\left(\left(\overrightarrow{\mathcal{P}^{i}}, \mathcal{Q}^{*}\right), \kappa^{i}\right)$ and that $\mathcal{Q}^{* *}$ is an initial segment of $\mathcal{M}_{\infty}(\mathcal{I})$. Then $\mathcal{Q}^{* *}$ is not $j$-bad.

Sketch. Let $\mathcal{I}$ and $\mathcal{Q}^{* *}$ be as in the statement of the lemma. We remark that there is no assumption on which model starts the main branch of $\mathcal{I}$. We must see that $\left(\left(W^{j}, \operatorname{ult}\left(\mathcal{Q}^{* *}, \pi_{i, j}, \kappa^{i}\right)\right), \kappa^{j}\right)$ is an iterable phalanx.

First, let $\mathcal{I}^{\prime}$ be the iteration tree on a phalanx with starting model $\mathcal{Q}^{j}$ and back-up models $\mathcal{N}_{\beta}$ for $\beta<\alpha^{i}$, that comes from $\mathcal{I}$ by the copyingenlarging-copying procedure done in the proof of Lemma 2.11. Let $\Phi$ be the map from $\mathcal{Q}^{* *}$ into an initial segment $\mathcal{M}$ of $\mathcal{M}_{\infty}\left(\mathcal{I}^{\prime}\right)$ that comes from this procedure. Then $\Phi \uparrow \kappa^{j}=\pi_{i, j} \uparrow \kappa^{j}$. So we have a map $\Psi$ with 
$\operatorname{crit}(\Psi) \geq \kappa^{j}$, and the following commutative diagram:

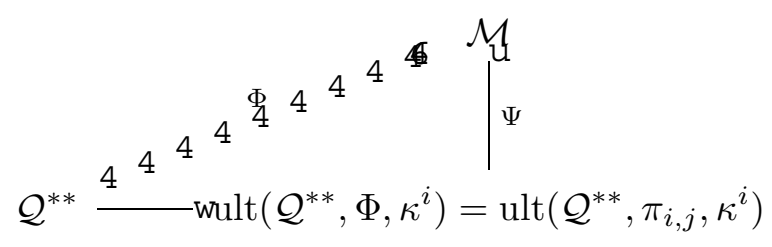

Now consider any $\gamma<\alpha^{j}$. Since $(4)_{\gamma}^{j}$ holds, we can apply [MiSchSt, 3.14] to get an iteration tree $\mathcal{U}_{\gamma}$ extending $\mathcal{T}^{j} \uparrow\left(\eta^{j}(\gamma)+1\right)$, and an elementary embedding $\psi_{\gamma}$ from $W^{j}$ into an initial segment $\mathcal{M}_{\gamma}$ of $\mathcal{M}_{\infty}\left(\mathcal{U}_{\gamma}\right)$ such that $\operatorname{crit}\left(\psi_{\gamma}\right) \geq \kappa_{\gamma}^{j}$. The following picture illustrates the situation:

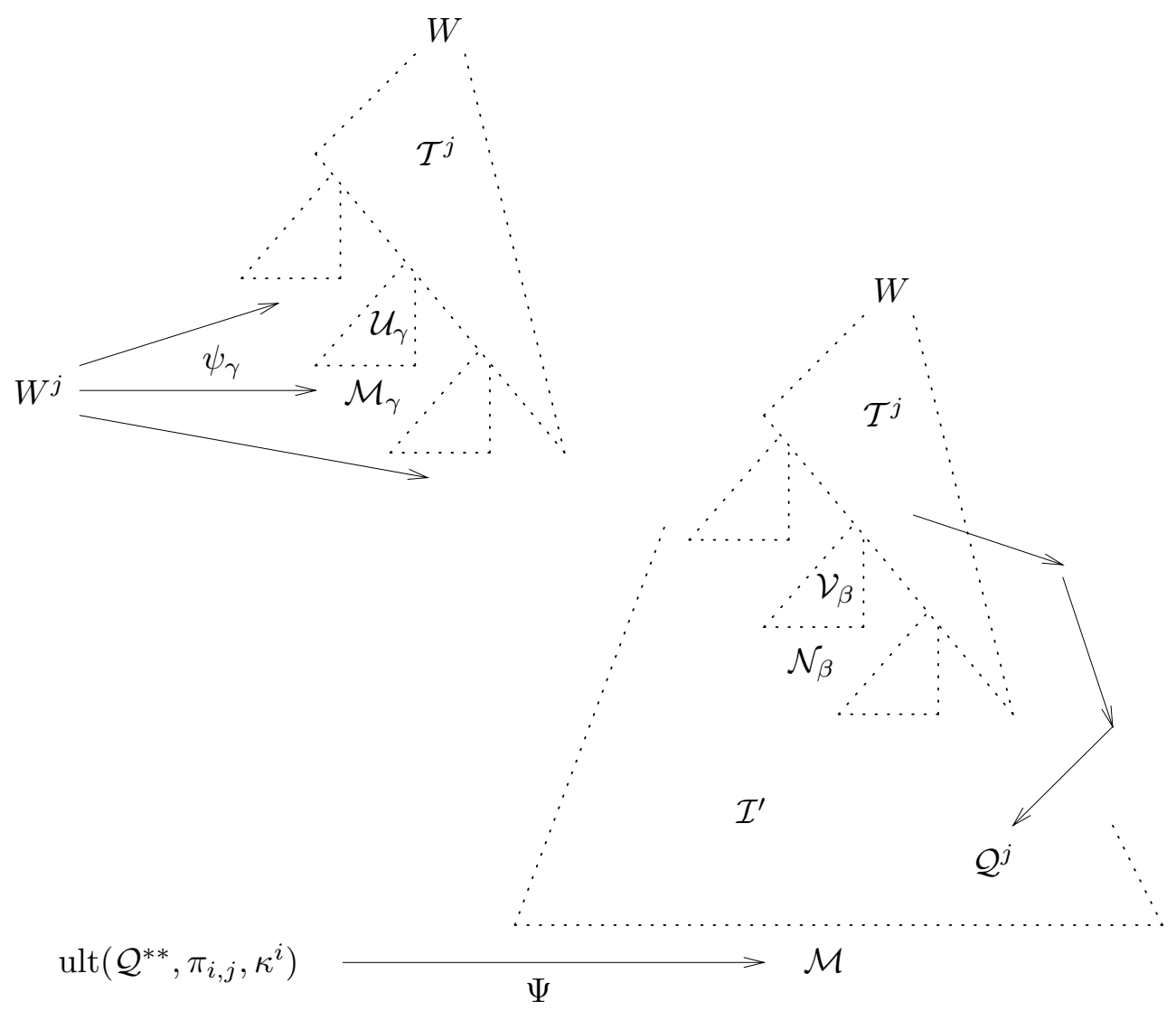

We can use the system of maps $\left(\left\langle\psi_{\gamma} \mid \gamma<\alpha^{j}\right\rangle, \Psi\right)$ to copy a putative iteration tree on $\left(\left(W^{j}, \operatorname{ult}\left(\mathcal{Q}^{* *}, \pi_{i, j}, \kappa^{i}\right)\right), \kappa^{j}\right)$ to a putative iteration tree on

$$
\left(\left(\left\langle\mathcal{M}_{\gamma} \mid \gamma<\alpha^{j}\right\rangle, \mathcal{M}\right), \vec{\lambda}^{j}\right)
$$


Fact 2.12.1 (Steel). $\left(\left(\left\langle\mathcal{M}_{\gamma} \mid \gamma<\alpha^{j}\right\rangle, \mathcal{M}\right), \vec{\lambda}^{j}\right)$ is iterable.

The fact is proved by the methods of [St, §9], and the lemma follows.

\section{A minimal $i$-good, $j$-bad premouse $\mathcal{M}^{*}$}

We continue the proof of Theorem 0.1. Our strategy is to find a premouse $\mathcal{M}^{*}$ which, like $\mathcal{Q}^{i}$, is both $i$-good and $j$-bad. Since $\mathcal{Q}^{*}$ is also $i$-good, we shall be able to coiterate the phalanxes $\left(\left(\overrightarrow{\mathcal{P}}^{i}, \mathcal{M}^{*}\right), \vec{\lambda}^{i}\right)$ and $\left(\left(\overrightarrow{\mathcal{P}}^{i}, \mathcal{Q}^{*}\right), \vec{\lambda}^{i}\right)$. We shall choose $\mathcal{M}^{*}$ so that this coiteration yields a map $\varphi$ from $\mathcal{M}^{*}$ into the last model on the $\mathcal{Q}^{*}$-side, with $\operatorname{crit}(\varphi) \geq \kappa^{i}$. Using the next lemma, and Lemma 2.12, we shall derive a contradiction.

Lemma 3.1. Let $\varphi: \mathcal{M} \longrightarrow \widetilde{\mathcal{M}}$ be an n-embedding with $\operatorname{crit}(\varphi) \geq \kappa^{i}$.

(a) If $\mathcal{M}$ is $\infty$-bad, then $\widetilde{\mathcal{M}}$ is $\infty$-bad.

(b) If $\mathcal{M}$ is $j$-bad, then $\widetilde{\mathcal{M}}$ is $j$-bad.

Proof. We prove (a), the proof of (b) being almost identical. Consider the diagram

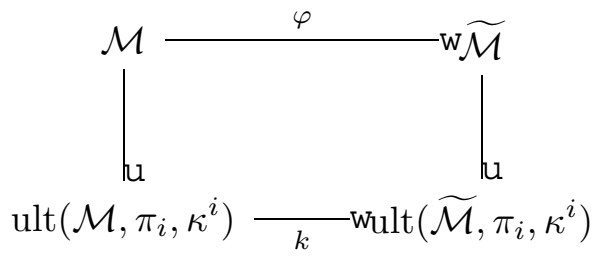

where

$$
k\left([a, f]_{E_{\pi_{i}}}^{\mathcal{M}}\right)=[a, \varphi(f)]_{E_{\pi_{i}}}^{\widetilde{\mathcal{M}}} .
$$

Then $k$ is an $n$-embedding with $\operatorname{crit}(k) \geq \kappa^{j}$ (in particular, $\operatorname{ult}\left(\widetilde{\mathcal{M}}, \pi_{i}, \kappa^{i}\right)$ is a premouse). So, a copying argument using the pair of maps (id $\uparrow W^{j}, k$ ) reduces the iterability of $\left(\left(W^{j}, \operatorname{ult}\left(\mathcal{M}, \pi_{i}, \pi_{i}\left(\kappa^{i}\right)\right), \kappa^{i}\right), \pi_{i}\left(\kappa^{i}\right)\right)$ to that of $\left(\left(W^{j}, \operatorname{ult}\left(\widetilde{\mathcal{M}}, \pi_{i}, \pi_{i}\left(\kappa^{i}\right)\right), \kappa^{i}\right), \pi_{i}\left(\kappa^{i}\right)\right)$.

Lemma 3.2. There is an $i$-good, $\infty$-bad premouse $\mathcal{M}^{*}$ with the following properties. Suppose that $\mathcal{I}$ is an iteration tree of successor length on $\left(\left(\overrightarrow{\mathcal{P}}^{i}, \mathcal{M}^{*}\right), \vec{\lambda}^{i}\right)$. Then

(a) No proper initial segment of $\mathcal{M}_{\infty}(\mathcal{I})$ is $\infty$-bad.

(b) Suppose that $\mathcal{M}_{\infty}(\mathcal{I})$ is $\infty$-bad. Then $\mathcal{M}_{\infty}(\mathcal{I})$ lies above $\mathcal{M}^{*}$ in the tree ordering of $\mathcal{I}$ and there is no dropping along the branch from $\mathcal{M}^{*}$ to $\mathcal{M}_{\infty}(\mathcal{I})$. That is, $\operatorname{root}^{\mathcal{I}}(\operatorname{lh}(\mathcal{I})-1)=\alpha^{i}$ and

$$
\mathcal{D}^{\mathcal{I}} \cap\left(\alpha^{i},(\operatorname{lh}(\mathcal{I})-1)\right)=\emptyset .
$$


Sketch. We define a sequence $\left\langle\mathcal{M}_{0}, \ldots, \mathcal{M}_{n}, \ldots\right\rangle$ by induction. Put $\mathcal{M}_{0}=$ $\mathcal{Q}^{i}$. Suppose that $\mathcal{M}_{n}$ has been defined, $\mathcal{M}_{n}$ is $i$-good, but there is an iteration tree $\mathcal{I}$ on $\left(\left(\overrightarrow{\mathcal{P}}^{i}, \mathcal{M}_{n}\right), \vec{\lambda}^{i}\right)$ witnessing that (a) or (b) fail for $\mathcal{M}_{n}$. Let $\mathcal{I}_{n}$ be such an iteration tree $\mathcal{I}$. Let $\mathcal{M}_{n+1}$ be the shortest initial segment $\mathcal{N}$ of $\mathcal{M}_{\infty}\left(\mathcal{I}_{n}\right)$ such that $\mathcal{N}$ is $\infty$-bad.

Fact 3.2.1(Steel). $\mathcal{M}_{n+1}$ is i-good.

Fact 3.2.2 (Steel). For some $n, \mathcal{M}_{n+1}$ is not defined.

Both facts are iterability properties of $W$ (recall that $\mathcal{M}_{0}=\mathcal{Q}^{i}$ is an initial segment of a model on $\mathcal{T}^{i}$, and that $\mathcal{T}^{i}$ is an iteration tree on $W$ ). They are proved by the methods of [St, $\S 9]$.

We remark that Lemma 3.2 expresses what we mean by "minimal" in the title of this section. Fix some premouse $\mathcal{M}^{*}$ as in Lemma 3.2. By elementarity, we may choose $\mathcal{M}^{*}$ so that $\mathcal{M}^{*} \in \operatorname{ran}\left(\pi_{j}\right)$; in fact, the proof of Lemma 3.2 gives such an $\mathcal{M}^{*}$. By Lemma $2.6, \mathcal{M}^{*}$ is also $j$-bad.

For the rest of this paper, let $(\mathcal{U}, \mathcal{V})$ be the pair of iteration trees resulting from the coiteration of $\left(\left(\overrightarrow{\mathcal{P}}^{i}, \mathcal{M}^{*}\right), \overrightarrow{\lambda^{i}}\right)$ versus $\left(\left(\overrightarrow{\mathcal{P}}^{i}, \mathcal{Q}^{*}\right), \vec{\lambda}^{i}\right)$.

Lemma 3.3. $\operatorname{root}^{\mathcal{U}}(\operatorname{lh}(\mathcal{U})-1)=\alpha^{i}$ and $\mathcal{D}^{\mathcal{V}} \cap\left(\alpha^{i},(\operatorname{lh}(\mathcal{V})-1)\right)=\emptyset$.

Proof. Suppose otherwise.

Case A. $\mathcal{Q}^{j}$ is a set premouse.

In Case A, we have available to us the $\kappa^{i}$-soundness of $\mathcal{Q}^{*}$. The usual fine structural considerations show that $\mathcal{Q}^{*}=\mathcal{M}_{\infty}(\mathcal{V})$ is an initial segment of $\mathcal{M}_{\infty}(\mathcal{U})$. Since $\mathcal{Q}^{*}$ is $\infty$-bad, Lemma 3.2(a) tells us that $\mathcal{Q}^{*}$ cannot be a proper initial segment of $\mathcal{M}_{\infty}(\mathcal{U})$. So $\mathcal{Q}^{*}=\mathcal{M}_{\infty}(\mathcal{U})$. Thus, $\mathcal{M}_{\infty}(\mathcal{U})$ is $\infty$-bad, contradicting Lemma 3.2(b).

Case B. $\mathcal{Q}^{j}$ is a weasel.

In Case B, we have available to us that $\mathcal{Q}^{*}$ is a model of ZFC. The usual fine structural considerations show that $\mathcal{M}_{\infty}(\mathcal{V})$ is an initial segment of $\mathcal{M}_{\infty}(\mathcal{U})$, and that $\operatorname{root}^{\mathcal{V}}(\operatorname{lh}(\mathcal{V})-1)=\alpha^{i}$. But then, by Lemma 3.1(a), $\mathcal{M}_{\infty}(\mathcal{V})$ is $\infty$-bad. By Lemma 3.2(a), we must have that $\mathcal{M}_{\infty}(\mathcal{V})=$ $\mathcal{M}_{\infty}(\mathcal{U})$. But this contradicts Lemma 3.2(b).

Lemma 3.4. $\mathcal{M}_{\infty}(\mathcal{U})$ is a initial segment of $\mathcal{M}_{\infty}(\mathcal{V})$. 
Proof. Otherwise, $\mathcal{M}_{\infty}(\mathcal{V})$ is a proper initial segment of $\mathcal{M}_{\infty}(\mathcal{U})$,

$$
\operatorname{root}^{\mathcal{V}}(\operatorname{lh}(\mathcal{V})-1)=\alpha^{i}
$$

and $\mathcal{D}^{\mathcal{V}} \cap\left(\alpha^{i},(\operatorname{lh}(\mathcal{V})-1)\right)=\emptyset$. But then, by Lemma $3.1(\mathrm{~b}), \mathcal{M}_{\infty}(\mathcal{V})$ is $\infty$-bad, which contradicts Lemma 3.2(a).

By lemma 3.1(b), we have that $\mathcal{M}_{\infty}(\mathcal{U})$ is $j$-bad. It follows easily that $\mathcal{M}_{\infty}(\mathcal{V})$ is $j$-bad. But, by Lemma $2.12, \mathcal{M}_{\infty}(\mathcal{V})$ is not $j$-bad. This contradiction completes the proof of Theorem 0.1.

\section{Acknowledgment}

The authors are grateful to John Steel for listening to their proof, and for his valuable comments.

\section{References}

[DeJe] K. I. Devlin and R. B. Jensen, Marginalia to a theorem of Silver, Logic Conference, Kiel 1974, Lec. Notes Math. 499, Springer, 1975, pp. 115-142.

[DoJe1] A. J. Dodd and R. B. Jensen, The Covering Lemma for K, Ann. Math. Logic 22 (1982), 1-30.

[DoJe2] — The Covering Lemma for L[U] , Ann. Math. Logic 22 (1982), 127155.

[Je] R. B. Jensen Non Overlapping Extenders, circulated notes.

[MaSt] D. A. Martin and J. R. Steel, Iteration Trees, J. Amer. Math. Soc. 7 (1994), $1-73$.

[Mi1] W. J. Mitchell. The Core Model for Sequences of Measures I, Math. Proc. Cambridge Phil. Soc. 95 (1984), 229-260.

[Mi2] _ The Core Model for Sequences of Measures II, unpublished.

[MiSchSt] W. J. Mitchell, E. Schimmerling, and J. R. Steel, The Covering lemma up to a Woodin cardinal, submitted to Ann. Pure Appl. Logic.

[MiSt] W. J. Mitchell and J. R. Steel, Fine Structure and Iteration Trees, Lec. Notes Logic 3, Springer, 1994.

[Sch1] E. Schimmerling, Combinatorial Principles in the Core Model for one Woodin Cardinal, Ann. Pure Appl. Logic 74 (1995), 153-201.

[Sch2] _ Successors of Weakly Compact Cardinals, preprint.

[St] J. R. Steel. The Core Model Iterability Problem, to appear in Lec. Notes Logic.

Department of Mathematics, University of Florida, Gainesville FL 32611

E-mail address: mitchell@math.ufl.edu

Department of Mathematics, Mit, Cambridge MA 02139

E-mail address: ernest@math.mit.edu 